\title{
Emergent universality in the two-neutron halo structure of ${ }^{22} \mathrm{C}$
}

\author{
L. A. Souza, ${ }^{1}$ E. Garrido, ${ }^{2}$ and T. Frederico ${ }^{1}$ \\ ${ }^{1}$ Instituto Tecnológico de Aeronáutica, DCTA, 12228-900, S. José dos Campos, Brazil \\ ${ }^{2}$ Instituto de Estructura de la Materia, IEM-CSIC, Serrano 123, E-28006 Madrid, Spain \\ (Received 8 July 2016; revised manuscript received 12 October 2016; published 9 December 2016)
}

\begin{abstract}
The structure of the two-neutron halo ${ }^{22} \mathrm{C}$ is investigated by means of a renormalized zero-range three-body model, with interactions in the $s$-wave channel, and a finite-range model with two- and three-body forces provided by the hyperspherical adiabatic expansion method. In both models the halo wave function in configuration space is obtained by using as inputs the two-body scattering lengths and the two-neutron separation energy. The halo-matter density is computed for ${ }^{22} \mathrm{C}$ with different three-body forces and low-energy parameters, with two-neutron separation energy within the range $50 \mathrm{keV} \leqslant S_{2 n} \leqslant 1000 \mathrm{keV}$. The halo-neutron density depends weakly on the neutron- ${ }^{20} \mathrm{C}$ scattering length as long as its absolute value is larger than the neutron-neutron one. The halo-neutron density is then analyzed by means of the root-mean-square radius, the probability density, and also the geometry, taking into account the angle between the two Jacobi coordinates. The results of finite-range and zero-range two-neutron-core models are compared. The effects in the halo structure of short-range and long-range three-body forces are studied, and the emergent universal behavior of the halo-neutron density and its geometry is pointed out.
\end{abstract}

DOI: 10.1103/PhysRevC.94.064002

\section{INTRODUCTION}

Since the pioneering work by Tanihata and coworkers [1], who identified the large matter tail in ${ }^{11} \mathrm{Li}$, the neutron drip line has been explored and expanded to other Borromean light nuclei (see, e.g., Ref. [2]). The interpretation of the neutronhalo formation was given in Ref. [3].

It is expected that at the neutron drip line or close to it, light neutron-halo nuclei can be formed. This is the case of the less-known neutron-rich carbon isotopes with the drip line sitting today on $N=16$. This corresponds to the ${ }^{22} \mathrm{C}$ nucleus, which shows the prominent property of having Borromean character; namely, none of its internal two-body subsystems, ${ }^{21} \mathrm{C}$ [4] or the neutron-neutron system, are bound. It is accepted that this nucleus forms a weakly bound two-neutron halo in $s$ waves [5]. However, its two-neutron separation energy $S_{2 n}$, which is an essential indicator of the presence of a halo, has not been firmly established yet. The mass evaluation [4] from 2003 has a large error and gives $S_{2 n}=0.4(8) \mathrm{MeV}$ and $S_{1 n}=0.3(6) \mathrm{MeV}$. A direct mass measurement lowered this value to $S_{2 n}=0.140(460) \mathrm{MeV}$ [6]. In Ref. [7], the unbound ${ }^{21} \mathrm{C}$ nucleus was investigated through single-proton removal in ${ }^{22} \mathrm{~N}$ at $68 \mathrm{MeV} / \mathrm{u}$, and the ${ }^{20} \mathrm{C}+$ neutron spectrum was described with an $s$-wave line shape with negative scattering length and $\left|a_{n-21}\right|<2.8 \mathrm{fm}$, suggesting $S_{2 n}<70 \mathrm{keV}$ (see also Ref. [8]). The NuDat 2.6 database provides the value of $S_{2 n}$ as $110 \pm 60 \mathrm{keV}$ [9].

A decade ago a theoretical work within a three-body model [10] predicted $S_{2 n}$ to be in the interval from 390 to $570 \mathrm{keV}$. The possibility of such a weakly bound halo in $s$ wave allows the neutrons to explore the classically forbidden region, where the presence of the short-range interaction is not so relevant for the three-body wave function. Furthermore, the properties of the low-energy parameters open the possibility to use concepts from the Efimov physics [11,12] already applied to cold atoms (see Refs. [13,14]), namely universality and model independence, which characterizes the large extension of the halo structure [15].

Experimental information on the structure of ${ }^{22} \mathrm{C}$ exists. The root-mean-square (rms) matter radius of $5.4 \pm 0.9 \mathrm{fm}$ was reported by Tanaka and collaborators [16], where the radius was extracted from the reaction cross section of ${ }^{22} \mathrm{C}$ on liquid hydrogen at $40 \mathrm{~A} \mathrm{MeV}$. They observed a large enhancement of the cross section compared to the neighbor nuclei ${ }^{19} \mathrm{C}$ and ${ }^{20} \mathrm{C}$. In addition, their analysis with a finite-range Glauber calculation under an optical-limit approximation showed that the two-valence neutrons occupy preferentially an $s_{1 / 2}$ orbital. The extracted rms matter radius was then used to constrain $S_{2 n}$ below $100 \mathrm{keV}$. This value was found in calculations with a renormalized three-body model [17], with a shell-model approach [18], within a cluster three-body model [19], with effective field theory [20], and with closed core calculations [21], which obtained $S_{2 n} \lesssim 0.3 \mathrm{MeV}$.

The core-recoil momentum distribution of ${ }^{22} \mathrm{C}$ was measured in Ref. [22] by observing the breakup of the halo on a carbon target at $240 \mathrm{MeV} /$ nucleon. A much narrower peak than in ${ }^{20} \mathrm{C}$ was observed, and a theoretical analysis of the measured cross sections and momentum distributions were performed by combining the eikonal reaction model for the single-neutron removal with psd shell-model calculations. Values for $S_{2 n}$ between 0.4 and $1.2 \mathrm{MeV}$ were used with an unbound level of ${ }^{21} \mathrm{C}$ at $0.3 \mathrm{MeV}$, and the halo neutrons in ${ }^{22} \mathrm{C}$ occupying an $s_{1 / 2}$ orbital. The comparison between the core-recoil distributions for ${ }^{22} \mathrm{C}$ and ${ }^{11} \mathrm{Li}$ [23] suggests similar sizes of their two-neutron halos (for further discussions, see Ref. [5]), and indirectly a halo binding somewhat larger than $100 \mathrm{keV}$. For comparison, we remind readers of the value of $369.15(65) \mathrm{keV}$ [24] for the ${ }^{11} \mathrm{Li}$ two-neutron separation energy. Recently, the ${ }^{20} \mathrm{C}$ core-recoil distribution data were analyzed within a Serber model [25] and a renormalized zero-range three-body model for the halo [26]. It was found that $100 \mathrm{keV} \lesssim S_{2 n} \lesssim 400 \mathrm{keV}$ 
reproduces the narrow peak in the momentum distribution, suggesting a more compact two-neutron halo.

Inspired by the three-body model proposed in Ref. [27], one relevant question is to check to what extent the Efimov universal physics, believed to play a role in the description of large halo systems, is actually supported by neutron-neutroncore models with finite-range two- and three-body potentials (with ranges compatible with the ${ }^{20} \mathrm{C}$ core). The three-body force is used to dial the two-neutron separation energy, and the two-body one is determined by the ${ }^{21} \mathrm{C}$ low-energy properties.

Our goal here is to investigate the structure of the twoneutron halo nucleus ${ }^{22} \mathrm{C}$ by means of a renormalized zerorange three-body model, which embody the Efimov physics, and a finite-range model with two- and three-body potentials. For that purpose, the halo-neutron density is studied in both models by means of the halo neutron root-mean-square radius, the neutron density, and the halo geometry. The aim is to establish the validity or limitations of universal scaling relations predicted by the zero-range model. These universal scaling relations are a consequence of the dominance of long-range Efimov potential over the properties of the halo [28-30], and they are expressed as model-independent correlations between low-energy observables $[13,15]$, where the short-range physics is parameterized by one three-body $s$-wave observable, like, e.g., the two-neutron separation energy.

Our computational tool to solve the eigenvalue equation for the neutron-neutron-core Hamiltonian with finite-range potential models relies on the hyperspherical adiabatic expansion method [31]. In the case of the renormalized zero-range model, subtracted integral equations (see Ref. [15]) are solved in momentum space and the configuration space halo wave function is then obtained [13].

This work is organized as follows. In Sec. II we briefly describe the hyperspherical adiabatic expansion method to solve the Hamiltonian eigenvalue equation for finite-range potentials. In Sec. III, the renormalized zero-range potential model and halo wave function are described. In Sec. IV the two- and three-body finite-range potentials and their parametrizations are given. In addition, we compare the correlation between $S_{2 n}$ and the halo-neutron rms radius for the finite-range and zero-range models. In Sec. $\mathrm{V}$ the spatial structure of the halo is investigated by analyzing scaling functions associated with the halo-neutron density and geometry using again the finite-range and zero-range models. We conclude our work in Sec. VI with the summary and the conclusions.

\section{FINITE-RANGE POTENTIAL MODEL}

The finite-range potential model used is this work is the hyperspherical adiabatic expansion method described in detail in Ref. [31]. In this method the three-body wave function is given in terms of the usual Jacobi coordinates $\{\boldsymbol{x}, \boldsymbol{y}\}$, from which the so-called hyper-radius $\rho\left(\rho^{2}=\sqrt{x^{2}+y^{2}}\right)$ and the five hyperangles $\Omega\left([\Omega] \equiv\left[\alpha, \Omega_{x}, \Omega_{y}\right]\right)$ are defined. The hyperangle $\alpha$ is given by $\tan \alpha=x / y$, and $\Omega_{x}$ and $\Omega_{y}$ give the directions of $\boldsymbol{x}$ and $\boldsymbol{y}$. Note that whereas the five hyperangles depend on the chosen Jacobi set, the hyper-radius does not.
In hyperspherical coordinates the three-body Hamiltonian $\hat{\mathcal{H}}$ takes the form

$$
\begin{aligned}
\hat{\mathcal{H}} & =-\frac{\hbar^{2}}{2 m} \hat{T}_{\rho}+\frac{\hbar^{2}}{2 m \rho^{2}} \hat{\Lambda}^{2}+\sum_{i<j} V_{i j}(\rho, \Omega) \\
& =-\frac{\hbar^{2}}{2 m} \hat{T}_{\rho}+\hat{\mathcal{H}}_{\Omega},
\end{aligned}
$$

where $\hat{T}_{\rho}=\frac{\partial^{2}}{\partial \rho^{2}}+\frac{5}{\rho} \frac{\partial}{\partial \rho}$ is the hyper-radial kinetic energy operator, $\hat{\Lambda}^{2}$ is the grand-angular operator (whose eigenfunctions are the hyperspherical harmonics), $V_{i j}$ gives the interaction between particles $i$ and $j$, and $m$ is the normalization mass used to define the Jacobi coordinates [31].

In the adiabatic expansion method the Schrödinger (or Faddeev) equation $(\hat{\mathcal{H}}-E) \Psi=0$ is solved in two steps. In the first one the angular eigenvalue problem

$$
\hat{\mathcal{H}}_{\Omega} \Phi_{n}(\rho, \Omega)=\frac{\hbar^{2}}{2 m} \frac{1}{\rho^{2}} \lambda_{n}(\rho) \Phi_{n}(\rho, \Omega)
$$

is solved for a set of fixed values of $\rho$. The set of angular eigenfunctions $\left\{\Phi_{n}(\rho, \Omega)\right\}$ form a complete basis, which is the one used in order to expand the total three-body wave function, i.e.,

$$
\Psi^{J M}(\boldsymbol{x}, \boldsymbol{y})=\frac{1}{\rho^{5 / 2}} \sum_{n} f_{n}(\rho) \Phi_{n}^{J M}(\rho, \Omega) .
$$

In a true calculation the angular eigenfunctions are expanded as

$$
\Phi_{n}^{J M}(\rho, \Omega)=\sum_{q} C_{q}^{(n)}(\rho)\left[\mathcal{Y}_{\ell_{x} \ell_{y}}^{K L}(\Omega) \otimes \chi_{s_{x} s_{y}}^{S}\right]^{J M},
$$

where $q$ collects all the quantum numbers $(q \equiv$ $\left.\left\{K, \ell_{x}, \ell_{y}, L, s_{x}, S\right\}\right), K=2 v+\ell_{y}+\ell_{y}$ is the hypermomentum, $\chi_{s_{x} s_{y}}^{S}$ is the spin function, and $\mathcal{Y}_{\ell_{x} \ell_{y}}^{K L M_{L}}(\Omega)$ are the usual hyperspherical harmonics.

The second step in the method corresponds to the calculation of the radial functions $f_{n}(\rho)$ in Eq. (3). They are given as the solution of the coupled set of radial equations:

$$
\begin{gathered}
\left\{-\frac{\partial^{2}}{\partial \rho^{2}}+\frac{2 m}{\hbar^{2}}\left[V^{(n)}(\rho)+V_{3 B}(\rho)\right]-\frac{2 m E}{\hbar^{2}}\right\} f_{n}(\rho) \\
-\sum_{n^{\prime}}\left\{2 P_{n n^{\prime}}(\rho) \frac{\partial}{\partial \rho}+Q_{n n^{\prime}}(\rho)\right\} f_{n^{\prime}}(\rho)=0
\end{gathered}
$$

where $E$ is the three-body energy, and the coupling functions $P_{n n^{\prime}}(\rho)$ and $Q_{n n^{\prime}}(\rho)$ can be found in Ref. [31].

The effective potentials $V^{(n)}(\rho)$ in Eq. (5) are the so-called adiabatic potentials, which are given by

$$
V^{(n)}(\rho)=\frac{\hbar^{2}}{2 m} \frac{\lambda_{n}(\rho)+\frac{15}{4}}{\rho^{2}},
$$

and where the $\lambda_{n}(\rho)$ functions are the eigenvalues of the angular equation (2).

Finally, it is well established that the use of bare two-body interactions usually leads to a three-body system that is marginally underbound or overbound compared to experimental values. To fine-tune the crucial three-body energy it 
is common to introduce a three-body interaction, which in Eq. (5) is denoted by $V_{3 B}(\rho)$. This is a phenomenological way of accounting for those polarizations of the particles that are beyond those described by the two-body interactions.

\section{ZERO-RANGE POTENTIAL MODEL}

The Hamiltonian for the zero-range model of the neutronneutron-core $(n-n-c)$ system is

$$
\hat{\mathcal{H}}_{z r}=-\frac{\hbar^{2}}{2 m_{\boldsymbol{r}_{x}}} \nabla_{\boldsymbol{r}_{x}}^{2}-\frac{\hbar^{2}}{2 m_{\boldsymbol{r}_{y}}} \nabla_{\boldsymbol{r}_{y}}^{2}+\sum_{i<j} \lambda_{i j} \delta\left(\boldsymbol{r}_{i}-\boldsymbol{r}_{j}\right),
$$

where two possible sets of the relative coordinates are defined in Figs. 4(a) and 4(b), and the associated reduced masses are represented by $m_{\boldsymbol{r}_{x}}$ and $m_{\boldsymbol{r}_{y}}$. The wave function is an eigenstate of the Hamiltonian $\left(\hat{\mathcal{H}}_{z r}+S_{2 n}\right) \Psi_{z r}=0$, where $S_{2 n}=-E$ is the two-neutron separation energy. The neutrons are supposed to be in a spin singlet state and the configuration space zerorange model wave function is [32]

$$
\begin{aligned}
\Psi\left(\boldsymbol{r}_{n}, \boldsymbol{r}_{n^{\prime}}\right)= & \int d \boldsymbol{q} \frac{e^{-\kappa_{n n}\left|\boldsymbol{r}_{n}-\boldsymbol{r}_{n^{\prime}}\right|}}{\left|\boldsymbol{r}_{n}-\boldsymbol{r}_{n^{\prime}}\right|} e^{i \boldsymbol{q} \cdot \boldsymbol{r}_{c, n n}} f_{n n}(\boldsymbol{q}) \\
& +\left\{\int d \boldsymbol{q} \frac{e^{-\kappa_{n c}\left|\boldsymbol{r}_{n}-\boldsymbol{r}_{c}\right|}}{\left|\boldsymbol{r}_{n}-\boldsymbol{r}_{c}\right|} e^{i \boldsymbol{q} \cdot \boldsymbol{r}_{n^{\prime}, n c}} f_{n c}(\boldsymbol{q})+\left(n \leftrightarrow n^{\prime}\right)\right\},
\end{aligned}
$$

where $\boldsymbol{r}_{n}, \boldsymbol{r}_{n^{\prime}}$, and $\boldsymbol{r}_{c}$ are the neutrons and core positions with respect to the center of mass of the $n-n-c$ system. The relative coordinate of the core to the neutron-neutron center of mass is $\boldsymbol{r}_{c, n n}$ and the relative coordinate of $n^{\prime}$ to the neutron-core center of mass is $\boldsymbol{r}_{n^{\prime}, n c}$. The $\kappa$ 's in the two-body subsystems wave functions are

$$
\begin{gathered}
\kappa_{n n}=\sqrt{2 \frac{\mu_{n n}}{\hbar^{2}}\left(S_{2 n}+\frac{\hbar^{2} q^{2}}{2 \mu_{n n, c}}\right)}, \\
\kappa_{n c}=\sqrt{2 \frac{\mu_{n c}}{\hbar^{2}}\left(S_{2 n}+\frac{\hbar^{2} q^{2}}{2 \mu_{n c, n}}\right)}
\end{gathered}
$$

with the reduced masses

$$
\begin{aligned}
& \mu_{n n}=\frac{m}{2}, \quad \mu_{n n, c}=m \frac{2 A}{A+2}, \\
& \mu_{n c}=m \frac{A}{A+1}, \quad \mu_{n c, n}=m \frac{A+1}{A+2},
\end{aligned}
$$

where $A$ is the mass number of the core, which for ${ }^{22} \mathrm{C}$ with a ${ }^{20} \mathrm{C}$ core is $A=20$. The zero-range wave function is an eigenstate of the Hamiltonian (7) and, with the exception of the configurations where two of the constituents overlap, the halo wave function is an eigenstate of the kinetic energy operator. In order to build the $s$-wave three-body wave function for the $n-n-c$ system, one needs to solve a coupled integral equation for the independent spectator functions $f_{n n}(\boldsymbol{q})$ and $f_{n c}(\boldsymbol{q})$. The formalism based on the renormalized zero-range three-body model leading to well-defined integral equations to compute the spectator functions from the $n n$ and $n c$ scattering lengths and the value of $S_{2 n}$ is presented and discussed in detail, for example, in Ref. [15].

\section{THREE-BODY PROPERTIES}

In order to construct the ${ }^{22} \mathrm{C}$ three-body wave function within the hyperspherical adiabatic expansion method, we use the Gaussian neutron-neutron interaction specified, for instance, in Ref. [33]. This potential contains central, spinspin, spin-orbit, and tensor parts, and it has been adjusted to reproduce the low-energy $n-n$ scattering data. In particular, it provides a neutron-neutron scattering length of $a_{n n}=$ $-18.4 \mathrm{fm}$. For the neutron-core interaction we use central and spin-orbit terms with a Woods-Saxon shape. Following Ref. [27], we choose the radius and diffuseness of the WoodsSaxon potentials equal to 1.25 and $0.65 \mathrm{fm}$, respectively.

For the $s$ waves the strength $V_{c}$ of the central potential is adjusted such that the lowest $s_{1 / 2}$ state in $n-{ }^{20} \mathrm{C}$ is unbound or barely bound. This is then a shallow potential not holding Pauli-forbidden states. In Table I we give the computed ${ }^{21} \mathrm{C}$ binding energy $E_{n c}$, scattering length $a_{n c}$, and effective range $r_{0}$, for different values of $V_{c}$. As we can see, any strength more attractive than $-21.0 \mathrm{MeV}$ makes the two-body neutron- ${ }^{20} \mathrm{C}$ system bound. The value of $V_{c}=-21.0 \mathrm{MeV}$ corresponds to basically infinite scattering length, or equivalently, a bound two-body state at zero energy. The calculation has been done taking the ${ }^{20} \mathrm{C}$ mass equal to $19.83 m$ where $m$ is the neutron mass. For $\ell>0$ waves we use the potential given in Ref. [27], where the strength of the central and spin-orbit potentials is taken equal to -42.0 and $-25.2 \mathrm{MeV}$, respectively.

As we can observe in Table I, the computed effective ranges are consistent with the ${ }^{20} \mathrm{C}$ root-mean-square (rms) matter radius of 2.98(5) fm [34]. That sizable effective range can in principle affect the universal correlations between observables in ${ }^{22} \mathrm{C}$. This point will be explored in the next sections, when the finite-range potentials will be used to compute the neutron halo distribution and the conditions under which the universal scaling laws are fulfilled will be investigated.

In Table II we show the results obtained for the two-neutron separation energy $S_{2 n}$ and the expectation value $\left\langle\rho^{2}\right\rangle$ when the two-body energy, $E_{n c}$, in the two-body neutron- ${ }^{20} \mathrm{C}$ system is put equal to zero $\left(V_{c}=-21.0 \mathrm{MeV}\right.$ in the $s$-wave neutron-core potential). Results including partial waves up to $\ell_{x}=\ell_{y}=8$ (left part) and with only $\ell_{x}=\ell_{y}=0$ waves (right part) are shown. The angular momenta $\ell_{x}$ and $\ell_{y}$ refer to the relative orbital angular momentum between the two particles connected by the Jacobi coordinate $\boldsymbol{x}$, and between the third particle and the center of mass of the first two. In the calculation a three-body force with a power $(P)$ dependence as given in Ref. [27] has been used:

$$
V_{3 B}^{P}(\rho)=\frac{V_{3 B}^{P}}{1+\left(\rho / \rho_{0}\right)^{3}} .
$$

TABLE I. Two-body binding energies $E_{n c}$, scattering lengths $a_{n c}$, and effective ranges $r_{0}$ for the $s$-wave $n-{ }^{20} \mathrm{C}$ system for different values the potential strength $V_{c}$.

\begin{tabular}{lccccr}
\hline \hline$V_{c}(\mathrm{MeV})$ & -21.0 & -25.0 & -30.0 & -32.0 & -33.5 \\
$E_{n c}(\mathrm{MeV})$ & $-1.5 \times 10^{-5}$ & -0.153 & -0.693 & -0.997 & -1.25 \\
$a_{n c}(\mathrm{fm})$ & 1192 & 13.4 & 6.98 & 6.03 & 5.51 \\
$r_{0}(\mathrm{fm})$ & 2.88 & 2.54 & 2.22 & 2.11 & 2.04 \\
\hline \hline
\end{tabular}


TABLE II. Computed values of $S_{2 n}$ (in MeV), $\left\langle\rho^{2}\right\rangle$ (in $\mathrm{fm}^{2}$ ), and $r_{n}$ (in fm), for different values of the three-body strength $V_{3 B}^{P}$, Eq. (11), with $\rho_{0}=5 \mathrm{fm}$ and for $V_{c}=-21.0 \mathrm{MeV}$. The left part of the table are the results including the components with $\ell_{x}, \ell_{y} \leqslant 8$, and the right part shows the results including only the $\ell_{x}=\ell_{y}=0$ components.

\begin{tabular}{ccccccccc}
\hline \hline$V_{3 B}^{P}$ & \multicolumn{3}{c}{$\ell_{x}, \ell_{y} \leqslant 8$} & & $V_{3 B}^{P}$ & \multicolumn{3}{c}{$\ell_{x}, \ell_{y}=0$} \\
\cline { 2 - 4 } \cline { 6 - 8 } & $S_{2 n}$ & $\left\langle\rho^{2}\right\rangle$ & $r_{n}$ & & $S_{2 n}$ & $\left\langle\rho^{2}\right\rangle$ & $r_{n}$ \\
\hline 0.0 & 1.83 & 25.16 & 3.54 & 0.0 & 1.52 & 28.06 & 3.75 \\
1.0 & 1.24 & 29.98 & 3.87 & & 1.0 & 0.954 & 35.31 & 4.20 \\
2.0 & 0.694 & 40.01 & 4.47 & & 2.0 & 0.447 & 54.10 & 5.20 \\
3.0 & 0.220 & 79.74 & 6.32 & & 2.5 & 0.230 & 83.79 & 6.47 \\
3.5 & 0.045 & 290.8 & 12.1 & & 3.0 & 0.060 & 249.4 & 11.2 \\
3.6 & 0.021 & 601.7 & 17.3 & & 3.1 & 0.035 & 407.4 & 14.3 \\
\hline \hline
\end{tabular}

For the future discussion, alternative shapes for the threebody potential will be also considered. In particular, we shall consider the Gaussian $(G)$ and exponential $(E)$ potentials:

$$
V_{3 B}^{G}(\rho)=V_{3 B}^{G} \mathrm{e}^{-\left(\rho / \rho_{0}\right)^{2}} \text { and } V_{3 B}^{E}(\rho)=V_{3 B}^{E} \mathrm{e}^{-\rho / \rho_{0}} .
$$

The results for different values of the strength $V_{3 B}^{P}$ and $\rho_{0}=5 \mathrm{fm}$ (as given in Ref. [27]) are also shown in the table. As we can see, without any three-body force the ${ }^{22} \mathrm{C}$ system is found to be bound, with two neutron separation energies 1.83 or $1.52 \mathrm{MeV}$, depending on the partial waves included in the calculation. Therefore, a repulsive three-body force is necessary in order to get a very weakly bound ${ }^{22} \mathrm{C}$ state.

The convergence of the results in Table II has been reached at two different levels. First, in the expansion of the angular eigenfunctions given in Eq. (4). This convergence is shown in Fig. 1, where the two lowest $\lambda_{n}(\rho)$ functions entering in the effective potentials (6) are plotted for different values of $K_{\max }$. As seen in the figure, a $K_{\max }$ value of 1600 is enough to get convergence up to a distance of at least $500 \mathrm{fm}$. This

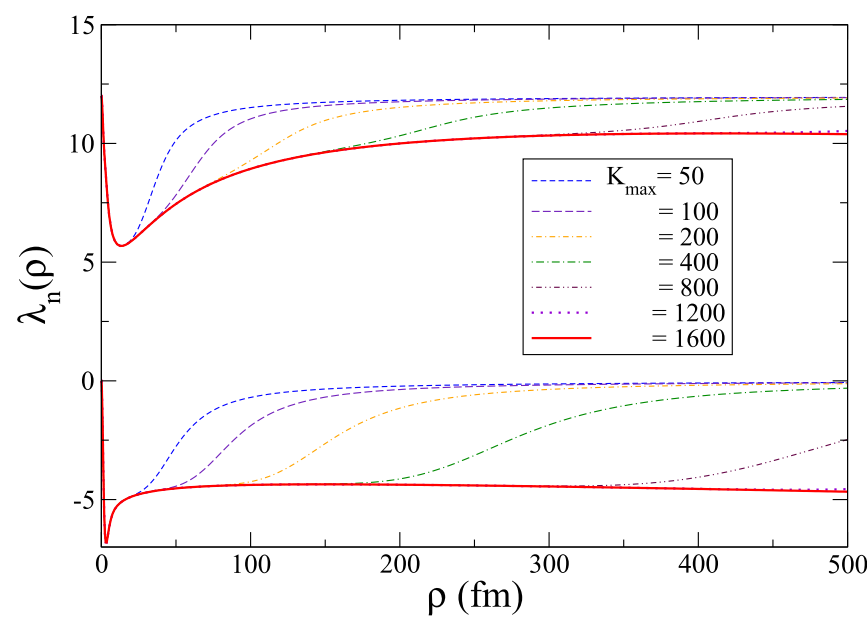

FIG. 1. Two lowest angular eigenvalue functions, $\lambda_{n}(\rho)$, for different values of $K_{\max }$ in the expansion in Eq. (4). The thick (red) solid line corresponds to $K_{\max }=1600$, which is the value used in the calculations.
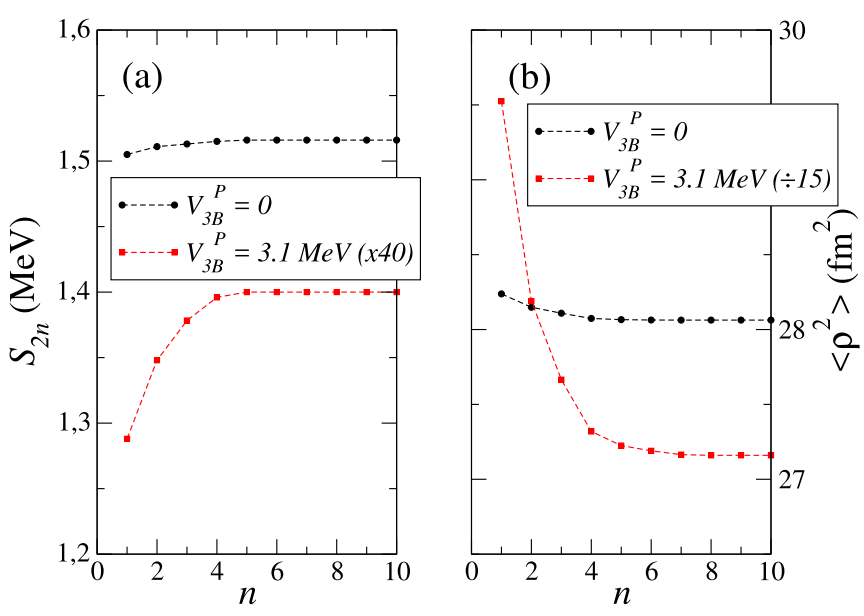

FIG. 2. Computed values of $S_{2 n}$ (a) and $\left\langle\rho^{2}\right\rangle$ (b) for the two states in Table II with $\ell_{x}=\ell_{y}=0$ and $V_{3 b}^{P}=0$ (black circles) and $V_{3 b}^{P}=3.1 \mathrm{MeV}$ (red squares). In the last case the results for $S_{2 n}$ have been multiplied by 40 and the ones of $\left\langle\rho^{2}\right\rangle$ have been divided by 15 .

distance is far enough to hold even the very weakly bound states given in Table II. In fact, for $S_{2 n}$ values of a few hundred $\mathrm{keV}$ and higher, the convergence of the adiabatic potentials up to $\rho=100 \mathrm{fm}$ is actually sufficient. In this work $K_{\max }=1600$ has been used.

The second convergence concerns the adiabatic expansion in Eq. (3). In Fig. 2 we show, as a function of the number of terms $n$ included in the expansion, the computed values of $S_{2 n}$ [Fig. 2(a)] and $\left\langle\rho^{2}\right\rangle$ [Fig. 2(b)] for the two states in Table II corresponding to $\ell_{x}=\ell_{y}=0$ and $V_{3 B}^{P}=0$ (circles) and $V_{3 B}^{P}=3.1 \mathrm{MeV}$ (squares). The last of these states corresponds to a very weakly bound and very extended system. For this reason, in order to have all the curves in the figure around the same scale, the computed values of $S_{2 n}$ and $\left\langle\rho^{2}\right\rangle$ for this case have been multiplied by 40 and divided by 15 , respectively. In this work a value of $n=10$ has been used, which, as seen in the figure, is enough to guarantee convergence of the results.

From [15] the quantity $r_{n}=\left\langle r_{n}^{2}\right\rangle^{1 / 2}$ is defined as

$$
r_{n}^{2}=\frac{22}{2}\left(R_{M}^{22} C\right)^{2}-\frac{20}{2}\left(R_{M}^{20} C\right)^{2},
$$

where $R_{M}^{22} C$ and $R_{M}^{20} C$ are the root-mean-square matter radii of ${ }^{22} \mathrm{C}$ and ${ }^{20} \mathrm{C}$, respectively.

The connection between $r_{n}$ and $\left\langle\rho^{2}\right\rangle$ can be easily obtained by remembering that for the three-body system ${ }^{22} \mathrm{C}(n+n+$ ${ }^{20} \mathrm{C}$ ) the matter radius is obtained as [35]

$$
\left(R_{M}^{22} C\right)^{2}=\frac{20}{22}\left(R_{M}^{20} C\right)^{2}+\frac{1}{22}\left\langle\rho^{2}\right\rangle,
$$

which leads to $r_{n}^{2}=\left\langle\rho^{2}\right\rangle / 2$. This result actually gives the neutron rms radius relative to the center of mass of the system for the case of an infinitely heavy core. For the present case of $A=20$ we will be content in comparing the $r_{n}$ results obtained within this approximation. The computed values of $r_{n}$ with the power three-body potential given in Eq. (11) are also given in Table II.

In Fig. 3(a) we show the two-neutron separation energies $S_{2 n}$ given in Table II versus the value of $r_{n}$. In the figure 

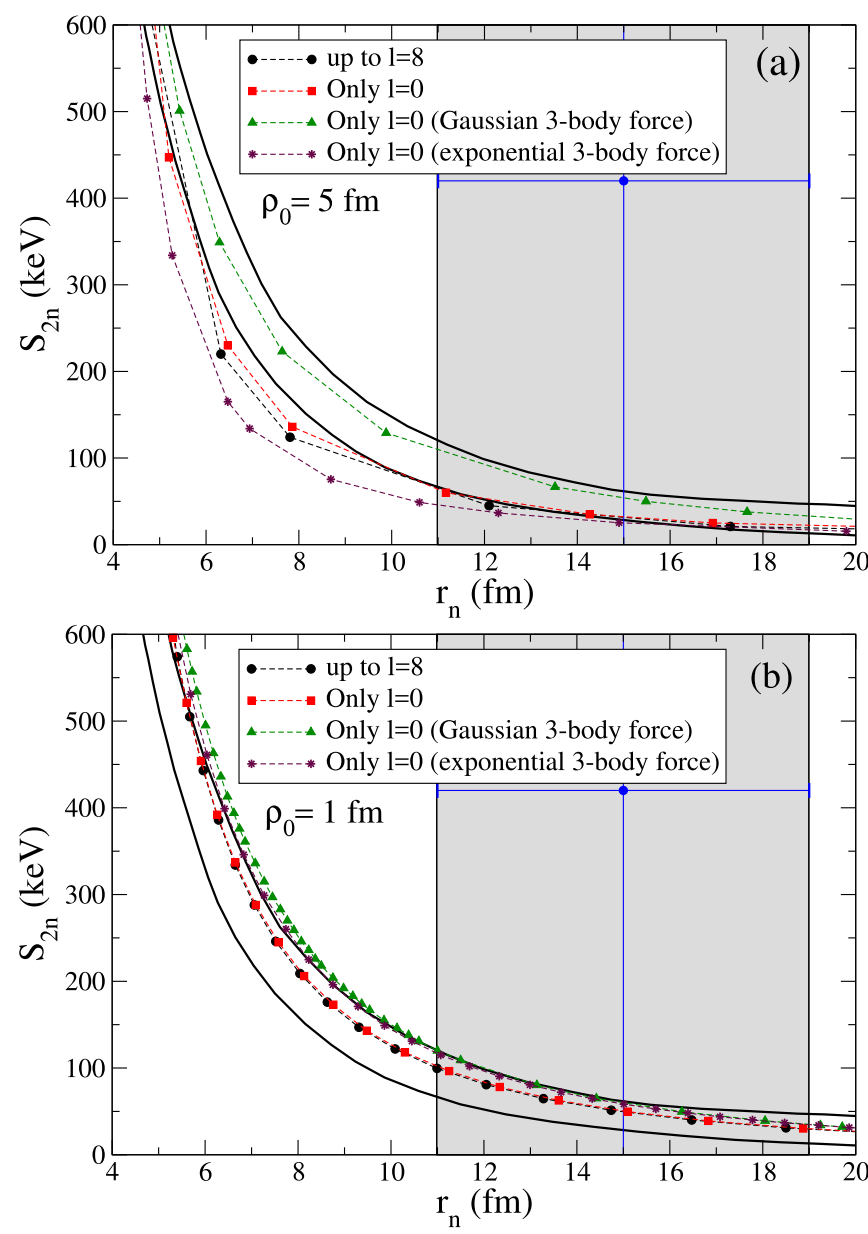

FIG. 3. Two-neutron separation energy $S_{2 n}$ as a function of $r_{n}$. The circles and the squares are the calculations including the $\ell_{x}, \ell_{y} \leqslant$ 8 components and the $\ell_{x}=\ell_{y}=0$ components, respectively. Panel (a): the solid curves are the ones shown in Fig. 22 of Ref. [15]. The triangles and the stars are the calculations with only $s$ waves but replacing the three-body force in Eq. (11) by a Gaussian and an exponential shape (12) (with $\rho_{0}=5 \mathrm{fm}$ ), respectively. Panel (b): the same as in panel (a) but for $\rho_{0}=1 \mathrm{fm}$ for the range of the three-body force. The point with error bars expresses the estimated ${ }^{22} \mathrm{C}$ neutron halo radius obtained in Ref. [17].

the circles show the results obtained with the $\ell_{x}, \ell_{y} \leqslant 8$ components, and the squares have been obtained including the $\ell_{x}=\ell_{y}=0$ components only. As we can see, the effect of the higher partial waves does not destroy the correlation between $S_{2 n}$ and $r_{n}$, and the scaling function, $S_{2 n}$ versus $r_{n}$, seems to show a quite small difference with respect to the $s$-wave case. This fact suggests that the effect of the higher partial waves can be associated with short-range physics accounted by the change of $S_{2 n}$, while other low-energy observables are correlated to this quantity.

A closer look into the scaling function can be taken by defining $r_{n}$ as done in Ref. [36]:

$$
r_{n}=\sqrt{\frac{\hbar^{2}}{m}} S_{2 n}^{-\frac{1}{2}} \mathcal{R}_{n}\left( \pm \varepsilon_{n c},-\varepsilon_{n n}, A\right)
$$

where the rescaled energies are

$$
\varepsilon_{n c}=\sqrt{\frac{\left|E_{n c}\right|}{S_{2 n}}}, \varepsilon_{n n}=\sqrt{\frac{\left|E_{n n}\right|}{S_{2 n}}},
$$

the $+/-$ signs refer to bound or virtual subsystems, and $A$ is the core mass number. The universal scaling function $\mathcal{R}_{n}$ is computed with the zero-range model, and it is the limit cycle of the correlation between the dimensionless quantities $r_{n} S_{2 n}^{\frac{1}{2}} \sqrt{m / \hbar^{2}}$ with the ratios $\varepsilon_{n c}$ and $\varepsilon_{n n}$ in the Efimov limit, namely when $E_{n c}$ and $E_{n n}$ tend to zero.

The scaling function (15) defines implicitly a universal correlation between $S_{2 n}$ and $r_{n}$ when the subsystem scattering lengths are fixed. This universal function is plotted in Fig. 3 for $E_{n c}=0$ (upper solid-black curve) and for the case where ${ }^{21} \mathrm{C}$ has a virtual state at $-100 \mathrm{keV}$ (lower solid-black curve). These two curves are actually shown in Fig. 22 of Ref. [15], where the universal function is obtained from the solution of the renormalized zero-range model described by Eq. (8) with a neutron-neutron virtual state energy of $-143 \mathrm{keV}$. As seen in the figure, the calculations done here seem to agree better with the lower solid curve than with the upper solid curve, which is the one corresponding to virtual states in ${ }^{21} \mathrm{C}$ at zero energy, which is also the case in the calculations shown by the circles and the squares. Therefore, a deviation from the universal curve computed with a zero-energy state of ${ }^{21} \mathrm{C}$ is observed, and in this case it becomes closer to the zero-range model results with $E_{n c}=-100 \mathrm{keV}$. This is because the repulsive three-body potential requires a more compact configuration for a given two-neutron separation energy, which is mimicked by increasing the absolute value of the ${ }^{21} \mathrm{C}$ virtual state energy in the zero-range model (see, e.g., Ref. [36]).

Our results are, however, sensitive to the shape of the threebody force. If the $V_{3 B}$ potential given in Eq. (11) is replaced by the Gaussian potential with $\rho_{0}=5 \mathrm{fm}$ in Eq. (12), our calculation gives rise to the curve shown in Fig. 3 by the triangles. This curve is clearly closer to the upper solid curve, namely the zero-range results with $E_{n c}=0$. However, if we take an exponential three-body force also with $\rho_{0}=5 \mathrm{fm}$, the result is shown by the stars in the figure, which is again closer to the zero-range calculation with the virtual state energy $E_{n c}=-100 \mathrm{keV}$.

This dependence on the shape of the three-body force, even for very small values of $S_{2 n}$, suggests that not only the inner part of the adiabatic potentials in Eq. (6) is modified by $V_{3 B}$, but also the tail of the potential changes. This is especially true for the power and exponential shapes of $V_{3 B}$, which die clearly more slowly than the Gaussian shape. Also, the value of the ${ }^{20} \mathrm{C}$ rms matter radius, 2.98(5) fm [34], suggests that the values of $\rho_{0}$ in the three-body force should be below $5 \mathrm{fm}$, since $\rho_{0}$ can be understood as the value of the hyper-radius obtained when the three particles are touching each other.

In Fig. 3(b) we show the same as in Fig. 3(a) but for $\rho_{0}=$ $1 \mathrm{fm}$. As we can see, when reducing the range all the curves tend to converge to the zero-range model results for $E_{n c}=0$. The fastest convergence appears when using a Gaussian threebody force, which we have observed that already for a range of $3 \mathrm{fm}$ agrees rather well with the zero-range results from Ref. [15]. This is consistent with the fact that the Gaussian 
three-body force dies faster than the others. Therefore, the tail of the adiabatic potentials given in Eq. (6), which for $E_{n c}=0$ and $s$ waves matches the universal Efimov potential, is left unchanged. We shall discuss this point more in detail in the next section.

\section{SPATIAL STRUCTURE}

\section{A. Halo-neutron density}

Let us introduce now the one-body density for the neutron as

$$
\eta\left(r_{y}\right)=r_{y}^{2} \int d \Omega_{y} d^{3} r_{x}|\Psi(\boldsymbol{x}, \boldsymbol{y})|^{2},
$$

which is normalized to 1 .

Since, as shown in Fig. 4, we have two possible sets of Jacobi coordinates, two different densities are therefore also possible. In order to compare with the zero-range model we choose the set of Jacobi coordinates given in Fig. 4(a), where $r_{y}$ is the relative distance of the neutron to the center of mass of the ${ }^{21} \mathrm{C}$ subsystem. In addition, to proceed with such a comparison, we introduce the density

$$
\eta_{n}\left(r_{n}\right)=\eta\left(r_{n} \frac{A+2}{A+1}\right),
$$

where $\boldsymbol{r}_{n}$ is the position of the neutron with respect to the center of mass of ${ }^{22} \mathrm{C}$.

In the limit of the zero-range potential the halo-neutron density can also be associated to a scaling function:

$$
\eta_{n}\left(r_{n}\right)=\sqrt{\frac{m}{\hbar^{2}}} S_{2 n}^{\frac{1}{2}} \mathcal{N}_{n}\left(\bar{r}_{n}, \pm \varepsilon_{n c},-\varepsilon_{n n}, A\right),
$$

where

$$
\bar{r}_{n}=\frac{m}{\hbar^{2}} S_{2 n}^{\frac{1}{2}} r_{n}
$$

The scaling function $\mathcal{N}_{n}$ is the limit cycle of the correlation between the dimensionless quantities $\eta_{n}\left(r_{n}\right) S_{2 n}^{-\frac{1}{2}} \sqrt{\hbar^{2} / m}$ with the ratios $\varepsilon_{n c}$ and $\varepsilon_{n n}$ in the Efimov limit, namely when $E_{n c}$ and $E_{n n}$ tend to zero.

We now compare the results obtained for $\eta_{n}\left(r_{n}\right)$ with the finite-range potentials and with the renormalized zero-range model, which allows us to build the scaling function (19). In this way it is possible to check the emergence of the scaling behavior of the density, as well as the limitations to model independence.
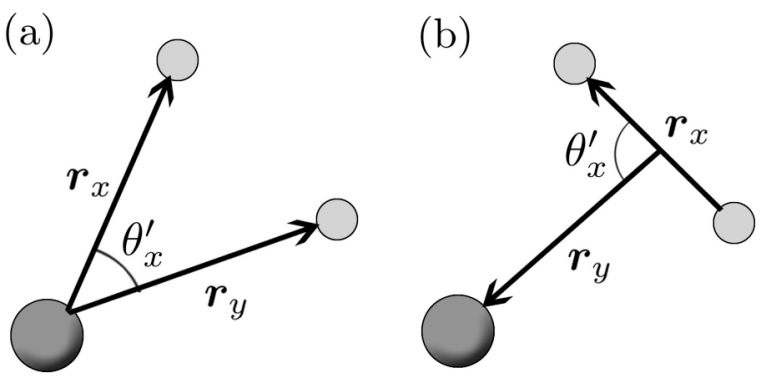

FIG. 4. Definition of the polar angles $\theta_{x}^{\prime}$ used for the two possible sets of Jacobi coordinates [panels (a) and (b)] in ${ }^{22} \mathrm{C}$.
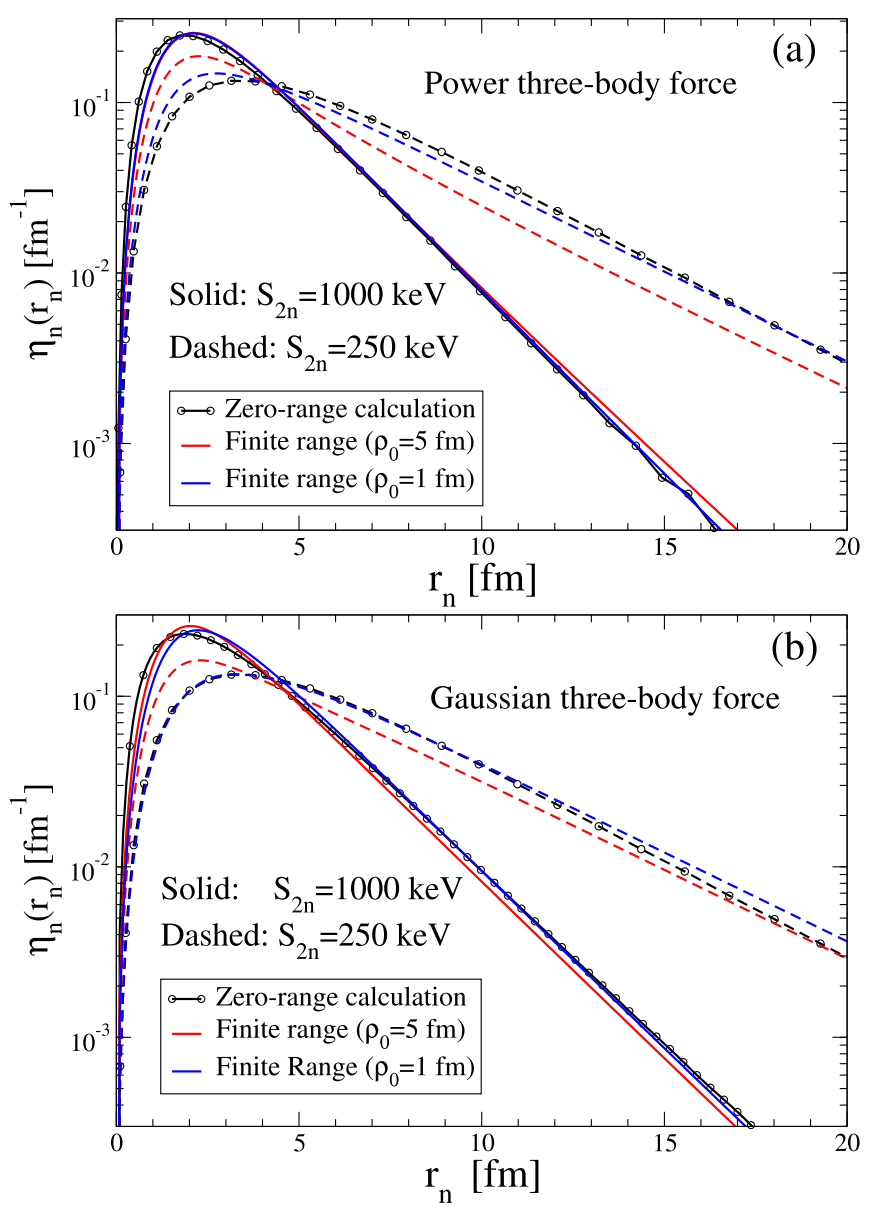

FIG. 5. Halo-neutron density $\eta_{n}\left(r_{n}\right)$ in ${ }^{22} \mathrm{C}$ with the powerdependent three-body potential (11) in panel (a) and the Gaussian three-body potential (12) in panel (b), compared to the zero-range model (represented by the black line with circles in both frames). Two values of $S_{2 n}$ are used for the calculations: $250 \mathrm{keV}$ (dashed curves) and $1000 \mathrm{keV}$ (solid curves). The energy of ${ }^{21} \mathrm{C}$ is fixed to $E_{n c}=0$. Two different ranges have been considered for the three-body force: $\rho_{0}=1 \mathrm{fm}$ (blue curves) and $\rho_{0}=5 \mathrm{fm}$ (red curves).

In a recent analysis of the ${ }^{22} \mathrm{C}$ core-recoil momentum distribution data [22], which were extracted from the breakup cross section in a carbon target at $240 \mathrm{MeV}$ per nucleon, the two-neutron separation energy $S_{2 n}$ was estimated to lie within the range $100 \mathrm{keV} \lesssim S_{2 n} \lesssim 400 \mathrm{keV}$ [26]. Therefore, for our considerations we shall use the values of $S_{2 n}=250 \mathrm{keV}$, in the middle of the above interval, and, in order to be well above the upper limit of the estimated range, we also choose $S_{2 n}=1000 \mathrm{keV}$. Furthermore, we use for the ${ }^{21} \mathrm{C}$ energy the value $E_{n c}=0$, representing large scattering lengths, which is not inconsistent with the analysis from Ref. [26]. In that work a small dependence on $E_{n c}$ in the core-recoil momentum distribution for a given $S_{2 n}$ was observed.

The results for the halo-neutron density are shown in Fig. 5. They have been obtained with the three-body force with a power dependence (11) [Fig. 5(a)] and with the Gaussian shape (12) [Fig. 5(b)]. We compare the finite-range potential neutron density to the one obtained from the zero-range model wave function (8). 

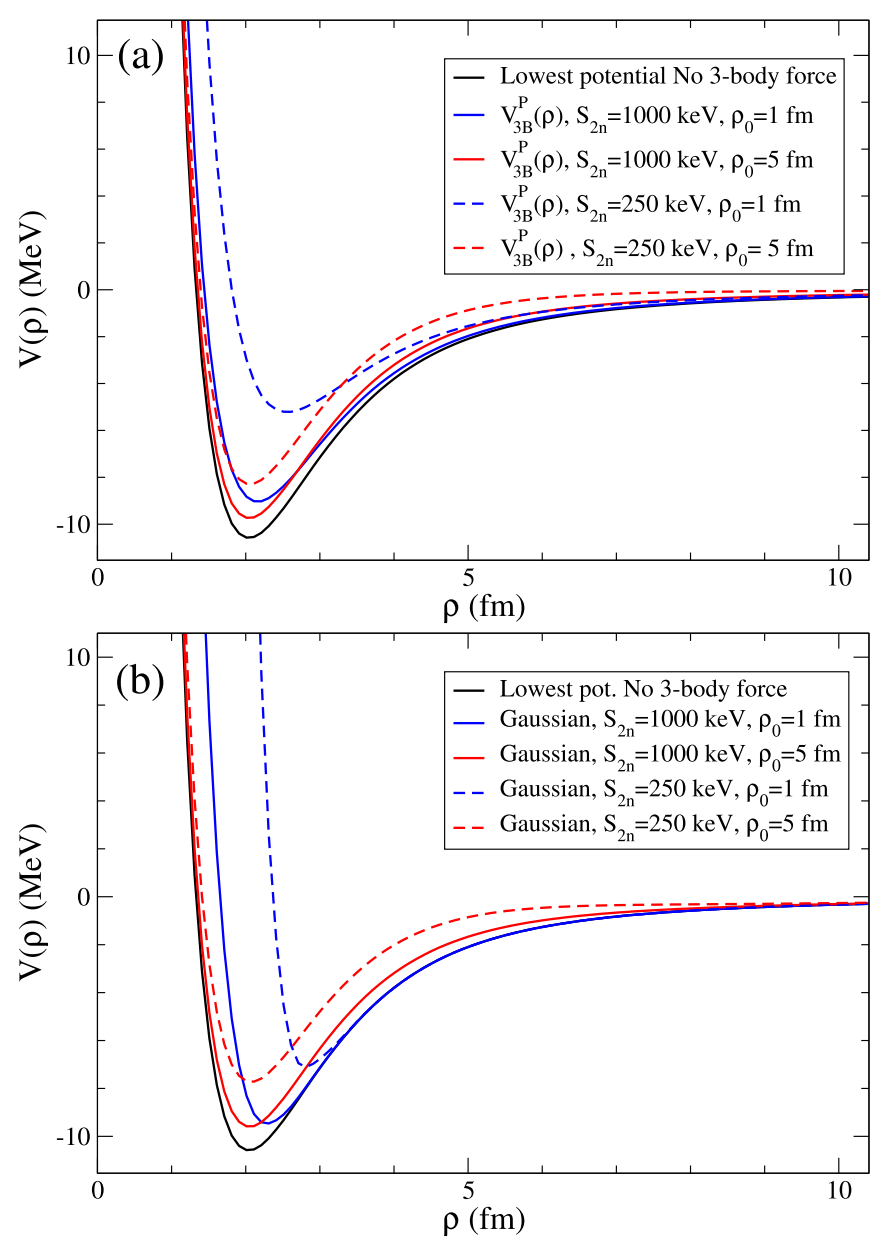

FIG. 6. Black curves: The lowest effective adiabatic potential $V(\rho)$ [as given in Eq. (6)] for ${ }^{22} \mathrm{C}$ when $E_{n c}=0$ and $\ell_{x}=\ell_{y}=0$. Solid curves: The lowest effective potential plus the three-body force producing a ${ }^{22} \mathrm{C}$ state with $S_{2 n}=1000 \mathrm{keV}$. Dashed curves: The lowest effective potential plus the three-body force producing a ${ }^{22} \mathrm{C}$ state with $S_{2 n}=250 \mathrm{keV}$. Two different ranges have been considered for the three-body force, $\rho_{0}=1 \mathrm{fm}$ (blue curves), and $\rho_{0}=5 \mathrm{fm}$ (red curves). In panel (a) the three-body force corresponds to the power dependence in Eq. (11), and in panel (b) the three-body force has the Gaussian shape given in Eq. (12).

Let us first consider the case with $S_{2 n}=1000 \mathrm{keV}$. The corresponding results are given in Fig. 5 by the solid curves. The results from the finite-range calculations are shown by the red and blue curves, which correspond to a range in the threebody potential of $\rho_{0}=5 \mathrm{fm}$ and $\rho_{0}=1 \mathrm{fm}$, respectively. The result from the zero-range model is given by the black-circle solid curve.

As we can see in Figs. 5(a) (three-body force with a power dependence) and 5(b) (three-body force with a Gaussian shape dependence), the finite-range results with the two chosen values of $\rho_{0}$ agree rather well with each other and with the zero-range model curve. In order to understand why this is happening, we should look at Fig. 6, where in both Figs. 6(a) and 6(b), the solid-black curve shows the lowest adiabatic potential as given in Eq. (6), corresponding to the ${ }^{22} \mathrm{C}$ nucleus with $E_{n c}=0$ and $\ell_{x}=\ell_{y}=0$. This means that this potential matches the universal Efimov potential, which is in fact found for distances of about $4 \mathrm{fm}$ and beyond. One should be aware that the zero-range model contains only the Efimov long-range potential damped at distances corresponding to the absolute value of the smallest scattering length, which is $a_{n n}=-17 \mathrm{fm}$. When the corresponding three-body force, power shape in Fig. 6(a) and Gaussian shape in Fig. 6(b), giving rise to $S_{2 n}=$ $1000 \mathrm{keV}$ with $\rho_{0}=1 \mathrm{fm}$ and $\rho_{0}=5 \mathrm{fm}$, is added, we then get the solid-blue and solid-red curves, respectively. As seen in the figure these two potentials agree reasonably well with the black effective potential which, as explained above, corresponds to the universal Efimov potential. This is particularly true for the case of $\rho_{0}=1 \mathrm{fm}$, whereas for $\rho_{0}=5 \mathrm{fm}$ the three-body force modifies the adiabatic potential up to larger distances. According to this, one would then expect a better agreement with the zero-range halo-neutron density in Fig. 5 in the case of $\rho_{0}=1 \mathrm{fm}$ (solid-blue curves), which in fact is observed in the figure. This result shows that the two-neutron halo is sufficiently weakly bound such that the effects of the details of the potentials at short distances fully disappear when $\rho_{0}=1 \mathrm{fm}$.

The same kind of analysis can be made when $S_{2 n}=$ $250 \mathrm{keV}$. In this case the corresponding curves in Figs. 5 and 6 are given by the dashed curves. When the three-body potential is given by the power three-body force the effective adiabatic potentials [dashed curves in Fig. 6(a)] change quite a lot from $\rho_{0}=1 \mathrm{fm}$ (blue-dashed) to $\rho_{0}=5 \mathrm{fm}$ (red-dashed). Furthermore, even if $\rho_{0}=1 \mathrm{fm}$, the three-body force modifies the tail of the pure adiabatic potential (black curve). As a result of this, the corresponding halo-neutron densities [dashed curves in Fig. 5(a)] are pretty different from each other, and even at large distances, also different from the zerorange calculation (especially when $\rho_{0}=5 \mathrm{fm}$ ), although both asymptotic tails are parallel to each other due to the same value of $S_{2 n}$ in both calculations. When the Gaussian three-body force is chosen, the corresponding effective potentials are again different from each other [dashed curves in Fig. 6(b)], but when $\rho_{0}=1 \mathrm{fm}$ (dashed-blue), the effective potential fully overlaps with the black curve for $\rho>3 \mathrm{fm}$. The consequence is that the halo-neutron density for $\rho_{0}=5 \mathrm{fm}$ [red-dashed curve in Fig. 5(b)] and $\rho_{0}=1 \mathrm{fm}$ (blue-dashed) are again different from each other, but the latter agrees now very well with the zero-range calculation shown by the black-dashed curve.

Summarizing, we have found that the only case that is not well reproduced by the zero-range model corresponds to $S_{2 n}=250 \mathrm{keV}$ and $\rho_{0}=5 \mathrm{fm}$. This is because the corresponding hyperspherical potential (red-dashed curve in Fig. 6) clearly deviates from the one obtained including only the short-range two-body forces (black curves) for $\rho$ values below $8 \mathrm{fm}$. The halo-neutron densities obtained for the other three cases are consistent with the zero-range model, and in this sense the density exhibits a universal behavior. This is, of course, related to the dominance of the Efimov tail of the hyperspherical potential observed in of Fig. 6, well represented by the hyperspherical potential obtained with the short-range neutron-core force model.

\section{B. Halo geometry}

We now investigate the geometry of the neutron-halo distribution by studying the dependence on the relative angle 
between the two Jacobi coordinates as depicted in Figs. 4(a) and 4(b). In order to make the visualization of the density easier, instead of using the angle $\theta_{x}^{\prime}$ we better introduce the coordinates $r_{x}^{\perp}=r_{x} \sin \theta_{x}^{\prime}$, which is the projection of $\boldsymbol{r}_{x}$ on a direction perpendicular to $\boldsymbol{r}_{y}$, and $r_{x}^{\|}=r_{x} \cos \theta_{x}^{\prime}$, which is the projection of $\boldsymbol{r}_{x}$ along $\boldsymbol{r}_{y}$. In this new set of coordinates, the volume element $d^{3} r_{x}$ can be written as

$$
d^{3} r_{x}=r_{x}^{\perp} d r_{x}^{\perp} d r_{x}^{\|} d \varphi_{x}^{\prime},
$$

and we can then define the density as

$$
F\left(r_{x}^{\perp}, r_{x}^{\|}\right)=\int r_{x}^{\perp} d \varphi_{x}^{\prime} d^{3} r_{y} \mid \Psi\left(\boldsymbol{x},\left.\boldsymbol{y}\right|^{2},\right.
$$

which is normalized to 1 after integration over $r_{x}^{\perp}$ and $r_{x}^{\|}$. This density can be, of course, obtained for any of the Jacobi coordinates described in Fig. 4, and gives a very intuitive feeling of the spatial distribution of the system.

More specifically, we have considered the ${ }^{22} \mathrm{C}$ wave function including partial waves with $\ell_{x}, \ell_{y} \leqslant 8$, and with a Gaussian three-body force with a range of $3 \mathrm{fm}$, close to the matter radius of the ${ }^{20} \mathrm{C}$ core. With this choice, the results for $S_{2 n}$ vs $r_{n}$ follow very closely the zero-range curve for $E_{n c}=0$ (see Fig. 3).

Our previous analysis of the halo-neutron density suggests that $F\left(r_{x}^{\perp}, r_{x}^{\|}\right)$should present a universal behavior close to the zero-range model results; namely it should be expressed by a scaling function (see Ref. [15]) in the limit of zero-range interaction as

$$
F\left(r_{x}^{\perp}, r_{x}^{\|}\right)=\frac{m}{\hbar^{2}} S_{2 n} \mathcal{F}\left(\bar{r}_{x}^{\perp}, \bar{r}_{x}^{\|}, \pm \varepsilon_{n c},-\varepsilon_{n n}, A\right)
$$

where the rescaled coordinates are

$$
\bar{r}_{x}^{\perp}=\frac{m}{\hbar^{2}} S_{2 n}^{\frac{1}{2}} r_{x}^{\perp}, \quad \bar{r}_{x}^{\|}=\frac{m}{\hbar^{2}} S_{2 n}^{\frac{1}{2}} r_{x}^{\|}
$$

the rescaled energies were defined in Eq. (16), and the \pm sign means bound/virtual subsystem. For the extreme case of $\varepsilon_{n c}=\varepsilon_{n n}=0$ the scaling function reduces to

$$
F\left(r_{x}^{\perp}, r_{x}^{\|}\right)=\frac{m^{2}}{\hbar^{4}} S_{2 n}^{-1} \mathcal{F}\left(\bar{r}_{x}^{\perp}, \bar{r}_{x}^{\|}, 0,0, A\right) .
$$

The scaling function $\mathcal{F}\left(\bar{r}_{x}^{\perp}, \bar{r}_{x}^{\|}, 0,0, A\right)$ is invariant under the scale transformation $S_{2 n} \rightarrow \lambda^{-2} S_{2 n}, r_{x}^{\perp} \rightarrow \lambda r_{x}^{\perp}$, and $r_{x}^{\|} \rightarrow$ $\lambda r_{x}^{\|}$, which means that the geometrical distribution should be preserved under this transformation, or when $S_{2 n}$ is changed. Such behavior should be found in a region of $r^{\prime}$ s smaller than $\left|a_{n n}\right|(17 \mathrm{fm})$, when $\left|a_{n c}\right|>\left|a_{n n}\right|$. This property is verified qualitatively by our calculations with the finite-range two- and three-body potentials for $r^{\prime}$ s smaller than $17 \mathrm{fm}$, as we are going to exemplify. Under this limitation, the geometry of the halo is universal and essentially determined by the ratio between the neutron and the core masses.

First we considered the Jacobi set shown in Fig. 4(a). The corresponding contour plots of the density $F$ defined in Eq. (22) are shown in Fig. 7 for four different two-neutron separation energies, 1000, 500, 250, and $50 \mathrm{keV}$. They are shown in Figs. 7(a), 7(b), 7(c), and 7(d), respectively. In all the cases the maximum of $F$ appears at an angle of about $\theta_{x}^{\prime}=50 \mathrm{deg}$, although obviously the system becomes more
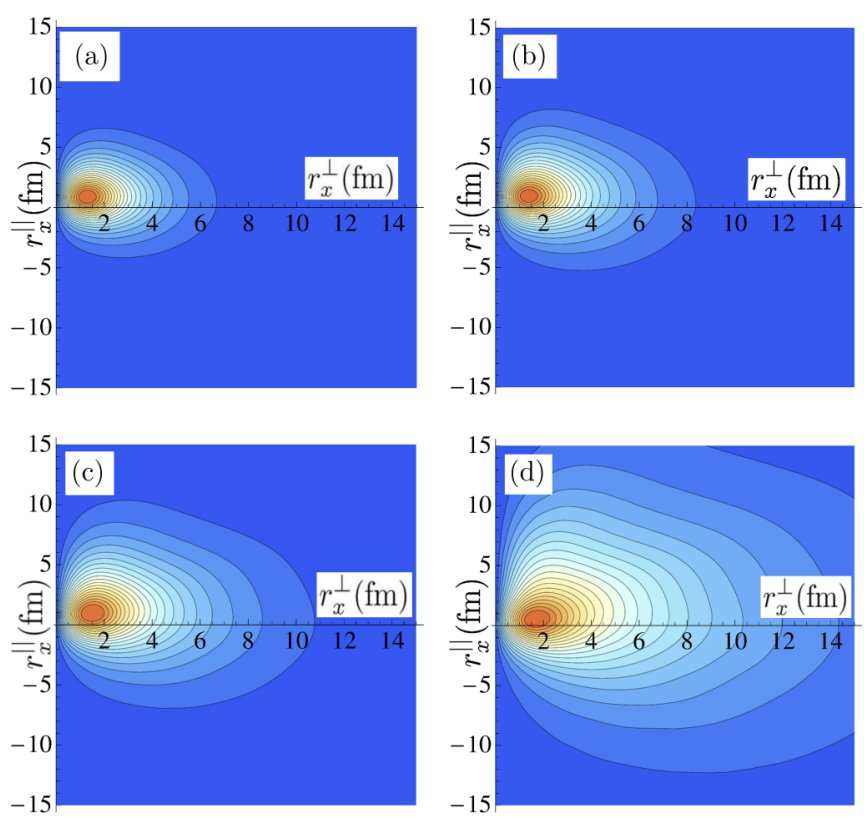

FIG. 7. Contour plot of the density $F\left(r_{x}^{\perp}, r_{x}^{\|}\right)$defined in Eq. (22) for the polar angle $\theta_{x}^{\prime}$ defined as in Fig. 4(a). Panels (a), (b), (c), and (d) correspond to a ${ }^{22} \mathrm{C}$ two-neutron separation energy of 1000, 500, 250 , and $50 \mathrm{keV}$, respectively.

spatially extended when the separation energy decreases. This universal behavior of the geometry shows in practice the dominance of the scaling property on the density distribution as expressed by the approximate validity of the scaling relation given in Eq. (25).

Note that the spatial structure shown in Fig. 7 is not symmetric with respect to the $r_{x}^{\|}=0$ axis. This is what it should be for a triangular distribution as the one shown in Fig. 4(a), which is completely different from a structure with a polar angle of $\pi-\theta_{x}^{\prime}$. However, for the Jacobi set shown in Fig. 4(b) the distribution, whose main features are similar to the ones shown in Fig. 7, is symmetric with respect to the $r_{x}^{\perp}$ axis. This arises from the fact that two neutrons are indistinguishable particles and the structures with polar angles $\theta_{x}^{\prime}$ and $\pi-\theta_{x}^{\prime}$ cannot be distinguished from each other.

\section{CONCLUSION}

The two-neutron halo structure of ${ }^{22} \mathrm{C}$ is investigated by means of a three-body (neutron-neutron-core) model with finite-range potentials, and with the renormalized zero-range model. The finite-range potential model calculations were performed in coordinate space by means of the hyperspherical adiabatic expansion method [31], and the zero-range model was treated by the subtraction method in momentum space [15]. In this second case the configuration space wave function was also computed.

The finite-range models and the zero-range one have the low-energy parameters, namely two-body scattering lengths and the two-neutron separation energy, fixed. In addition, besides the Wood-Saxon neutron $-{ }^{20} \mathrm{C}$ potential from Ref. [27], we have considered a repulsive three-body force in order 
to be able to study the possibility of a very weakly bound two-neutron halo to the core.

As suggested by a recent analysis of the ${ }^{22} \mathrm{C}$ core-recoil momentum distribution data [22], we focus our study within the two-neutron separation energy range $100 \mathrm{keV} \lesssim S_{2 n} \lesssim$ $400 \mathrm{keV}$ [26]. We have then investigated the halo structure for binding energies between 50 and $1000 \mathrm{keV}$.

Three scaling functions associated with the neutron halo structure of ${ }^{22} \mathrm{C}$ were addressed: (i) the correlation between $S_{2 n}$ and $r_{n}$ (the rms radius of the neutron with respect to the center of mass), (ii) the halo-neutron density, and (iii) the halo geometry considering the angle between the two Jacobi coordinates. We have verified that the halo-neutron probability density depends weakly on the neutron $-{ }^{20} \mathrm{C}$ scattering length as long as its absolute value is larger than the neutron-neutron one.

The results of the finite-range and zero-range models have been compared to check the emergence of the universal scaling relations, taking advantage of different three-body forces and range values. For two-body potential domination, which happens for $S_{2 n}=1000 \mathrm{keV}$ with different three-body potentials, the neutron density approaches the scaling function from the zero-range model. In this case the effective range is $2.88 \mathrm{fm}$, which seems not large enough to make the finite range potential results depart from the zero-range model ones.

The decrease of the two-neutron separation energy in our model is done by enhancing the repulsive three-body force. Even when $S_{2 n}=250 \mathrm{keV}$, for a range of the three-body force of $5 \mathrm{fm}$ the departure from the universal zero-range function starts to be noticeable. This deviation is understood from the difference between the lowest hyper-radial potentials with and without three-body force, since the latter fulfills the Efimov conditions. Differently, for a range in the three-body force equal to $1 \mathrm{fm}$, all the three-body potentials produce modelindependent halo densities close to the zero-range model.

The geometry of the halo has been investigated by exploring the angular dependence of the neutron density with a Gaussian three-body force with a range of $3 \mathrm{fm}$ (close to the ${ }^{20} \mathrm{C}$ observed matter radius), and with $S_{2 n}$ between 50 and $1000 \mathrm{keV}$. In the unitary limit, namely for infinite scattering length, the scaling function expressing such density says that the spatial distribution of the neutron should scale with $1 / \sqrt{S_{2 n}}$, whereas its shape and angular structure should be preserved. This scaling property has been observed.

The emergence of the universal behavior in the halo-neutron density distribution and geometry, within the present model consistent with scaling laws found in the zero-range interaction limit and dominated by the universal Efimov physics, is a robust outcome in the case of the two-neutron halo in ${ }^{22} \mathrm{C}$ for force ranges up to about the size of of the core.

\section{ACKNOWLEDGMENTS}

We are thankful for partial support from the Brazilian agencies Fundação de Amparo à Pesquisa do Estado de São Paulo (FAPESP), Conselho Nacional de Desenvolvimento Científico e Tecnológico $(\mathrm{CNPq})$ and Coordenação de Aperfeiçoamento de Pessoal de Nível Superior (CAPES). This work was supported by funds provided by Dirección General de Investigación Científica (DGI) of Ministerio de Economía, Industria y Competitividad (MINECO) (Spain) under Contract No. FIS2014-51971-P and the Vicepresidencia de Investigación Científica y Técnica (VICyT) (Consejo Superior de Investigaciones Científicas (CSIC)) under Project i-LINK 1056.
[1] I. Tanihata et al., Phys. Rev. Lett. 55, 2676 (1985).

[2] I. Tanihata, H. Savajols, and R. Kanungo, Prog. Part. Nucl. Phys. 68, 215 (2013).

[3] P. G. Hansen and B. Jonson, Europhys. Lett. 4, 409 (1987).

[4] G. Audi, A. H. Wapstra, and C. Thibault, Nucl. Phys. A 729, 337 (2003).

[5] K. Riisager, Phys. Scr. T 152, 014001 (2013).

[6] L. Gaudefroy, W. Mittig, N. A. Orr, S. Varet, M. Chartier, P. Roussel-Chomaz, J. P. Ebran, B. Fernández-Domínguez, G. Frémont, P. Gangnant, A. Gillibert, S. Grévy, J. F. Libin, V. A. Maslov, S. Paschalis, B. Pietras, Y.-E. Penionzhkevich, C. Spitaels, and A. C. C. Villari, Phys. Rev. Lett. 109, 202503 (2012).

[7] S. Mosby et al., Nucl. Phys. A 909, 69 (2013).

[8] R. B. Firestone, Nucl. Data Sheets 127, 1 (2015).

[9] http://www.nndc.bnl.gov/chart/reColor.jsp?newColor=s2n.

[10] W. Horiuchi and Y. Suzuki, Phys. Rev. C 74, 034311 (2006).

[11] V. Efimov, Phys. Lett. B 33, 563 (1970).

[12] V. Efimov, Few-Body Syst. 51, 79 (2011).

[13] T. Frederico, L. Tomio, A. Delfino, M. R. Hadizadeh, and M. T. Yamashita, Few-Body Syst. 51, 87 (2011).

[14] F. Ferlaino, A. Zenesini, M. Berninger, B. Huang, H.-C. Nägerl, and R. Grimm, Few-Body Syst. 51, 113 (2011).
[15] T. Frederico, M. T. Yamashita, A. Delfino, and L. Tomio, Prog. Part. Nucl. Phys. 67, 939 (2012).

[16] K. Tanaka, T. Yamaguchi, T. Suzuki, T. Ohtsubo, M. Fukuda, D. Nishimura, M. Takechi, K. Ogata, A. Ozawa, T. Izumikawa, T. Aiba, N. Aoi, H. Baba, Y. Hashizume, K. Inafuku, N. Iwasa, K. Kobayashi, M. Komuro, Y. Kondo, T. Kubo, M. Kurokawa, T. Matsuyama, S. Michimasa, T. Motobayashi, T. Nakabayashi, S. Nakajima, T. Nakamura, H. Sakurai, R. Shinoda, M. Shinohara, H. Suzuki, E. Takeshita, S. Takeuchi, Y. Togano, K. Yamada, T. Yasuno, and M. Yoshitake, Phys. Rev. Lett. 104, 062701 (2010).

[17] M. T. Yamashita, R. M. de Carvalho, T. Frederico, and L. Tomio, Phys. Lett. B 697, 90 (2011); 715, 282 (2012).

[18] H. T. Fortune and R. Sherr, Phys. Rev. C 85, 027303 (2012).

[19] S. N. Ershov, J. S. Vaagen, and M. V. Zhukov, Phys. Rev. C 86, 034331 (2012).

[20] B. Acharya, C. Ji, and D. R. Phillips, Phys. Lett. B 723, 196 (2013); B. Acharya and D. R. Phillips, EPJ Web of Conferences 113, 06013 (2016).

[21] T. Suzuki, T. Otsuka, C. Yuan, and N. Alahari, Phys. Lett. B 753, 199 (2016).

[22] N. Kobayashi et al., Phys. Rev. C 86, 054604 (2012).

[23] I. Tanihata, J. Phys. G 22, 157 (1996). 
[24] M. Smith, M. Brodeur, T. Brunner, S. Ettenauer, A. Lapierre, R. Ringle, V. L. Ryjkov, F. Ames, P. Bricault, G. W. F. Drake, P. Delheij, D. Lunney, F. Sarazin, and J. Dilling, Phys. Rev. Lett. 101, 202501 (2008).

[25] R. Serber, Phys. Rev. 72, 1008 (1947).

[26] L. A. Souza, F. F. Bellotti, T. Frederico, M. T. Yamashita, and L. Tomio, Phys. Lett. B 757, 368 (2016); Few-Body Syst. 57, 361 (2016).

[27] Y. Kucuk and J. A. Tostevin, Phys. Rev. C 89, 034607 (2014).

[28] A. S. Jensen, K. Riisager, D. V. Fedorov, and E. Garrido, Rev. Mod. Phys. 76, 215 (2004).

[29] E. Braaten and H.-W. Hammer, Phys. Rep. 428, 259 (2006).
[30] N. T. Zinner and A. S. Jensen, J. Phys. G: Nucl. Part. Phys. 40, 053101 (2013).

[31] E. Nielsen, D. V. Fedorov, A. S. Jensen, and E. Garrido, Phys. Rep. 347, 373 (2001).

[32] T. Frederico, Few-Body Syst. 55, 651 (2014).

[33] E. Garrido and. E. Moya de Guerra, Nucl. Phys. A 650, 387 (1999).

[34] A. Ozawa et al., Nucl. Phys. A 691, 599 (2001).

[35] D. V. Fedorov, E. Garrido, and A. S. Jensen, Phys. Rev. C 51, 3052 (1995).

[36] M. T. Yamashita, L. Tomio, and T. Frederico, Nucl. Phys. A 735, 40 (2004). 